This document has two parts:

The original preprint of "How to Build an Infinite Lottery Machine"

And

Preprint, co-authored with Alexander R. Pruss, of Correction to John D. Norton “How to Build an Infinite Lottery Machine." It corrects an error in Section 11.3 of the original preprint. 


\title{
How to Build an Infinite Lottery Machine
}

\author{
John D. Norton ${ }^{1}$ \\ Department of History and Philosophy of Science \\ University of Pittsburgh \\ http://www.pitt.edu/ jdnorton
}

\begin{abstract}
An infinite lottery machine is used as a foil for testing the reach of inductive inference, since inferences concerning it require novel extensions of probability. Its use is defensible if there is some sense in which the lottery is physically possible, even if exotic physics is needed. I argue that exotic physics is needed and describe several proposals that fail and at least one that succeeds well enough.
\end{abstract}

\section{Introduction}

An infinite lottery machine, as I shall construe it here, chooses from a countable infinity of outcomes $n=1,2,3, \ldots$ without favoring any outcome. The machine is immediately problematic if one applies routine probabilistic notions to it. If $P(n)$ is the probability of outcome $n$, then the probability that one of the outcomes is realized is given by the infinite sum:

$$
P(1)+P(2)+\ldots+P(n)+\ldots=1
$$

It must equal one, as shown, since one of the outcomes $1,2,3, \ldots$ is sure to happen. However each individual outcome must have the same probability

$$
\varepsilon=P(1)=P(2)=\ldots=P(n)=\ldots
$$

If $\varepsilon>0$, then the infinite sum of probabilities in (1) is infinite, contradicting the axiom of probability theory that the probability of the whole event space is unity. If however we set $\varepsilon=0$, then the same sum yields zero.

\footnotetext{
${ }^{1}$ I thank John Earman, Casper Storm Hansen, Bryan Roberts, David Snoke, Teddy Seidenfeld and Porter Williams for helpful discussion.
} 
The infinite lottery is almost always conceived in the philosophical literature as a technical problem for probabilistic inference. On the evidence of the design and set up of the machine, what inductive support does each outcome accrue ${ }^{2}$ Because of the problem above, a probability seems unable to measure that support. Most attempts to escape adjust the notion of probability. De Finetti $(1972$; §5.17) discarded "countable additivity," the capacity to sum the infinitely many outcomes of (1) to yield a probability. Instead, he employs "finite additivity," which allows only finitely many terms in (1) to be summed. Then we can set $\varepsilon=0$ in (2) without immediate problems, since the infinite sum of (1) is no longer admissible as a means of computing a probability. There has been considerable critical weighing of the ramifications of discarding countable additivity. See, for example, Bartha (2004), Blackwell and Diaconis (1996), Kadane, Schervish, and Seidenfeld (1986), Kadane and O'Hagan (1995) and Williamson (1999). A more elaborate proposal, sets $\varepsilon$ in (2) to an infinitesimal, employing non-standard analysis. See for example Benci, Horsten, and Wenmackers (2013) and Wenmackers and Horsten, (2013). The cogency of this approach has in turn been debated. See for example, Pruss (2014), Williamson (2007) and Weintraub (2008).

My primary goal in this paper is not to address these technical problems in probabilistic inference. Rather it is to address a physical problem: is an infinite lottery machine physically possible? If, as some contend, the very idea of the lottery is internally contradictory, then it is a physical impossibility and we should renounce it as a probe of our notions of probabilistic inference.

This simple-sounding question of physical possibility will prove to be treacherous. No one, as far as I know, has found a way to build a real, infinite lottery machine. Instead, time and again, obvious proposals for such machines run into fatal difficulties. Section 2 below reviews a few natural proposals to give a sense of how they fail. The repeated failures suggest some principled obstacle. In Section 3, two arguments that try to establish the impossibility of infinite lottery machines are shown to fail.

If nothing rules out an infinite lottery machine in principle, but none are constructible in our ordinary world, then in just what sense could one be said to be physically possible? Here we

\footnotetext{
${ }^{2}$ Here I will restrict myself to objective approaches to inductive inference. Analogous problems arise if we ask what our subjective belief should be in each outcome.
} 
run headlong into the awkward problem that we have no precise notion of physical possibility. Rather physical possibility comes in a myriad of varieties. Stricter forms allow only minor departures from actuality. More exotic forms skirt contradictions, such as supertask systems, in which an infinity of distinct actions are completed in finite time.

Since there is no single, universally correct notion of physical possibility, I propose in Section 4 that we choose a sense of physical possibility fitting to the infinite lottery's role as a probe or foil for inductive inference. This automatically authorizes a quite liberal notion of physical possibility. For we expect that inductive reasoning, like deductive reasoning, should be possible not just in worlds exactly like ours, but in any world that (a) can be described without logical contradiction (hence non-trivial deductive inference is possible) and (b) is governed by some regularities (so inductive inference is possible).

The sections following map out suitable regularities conservatively. This is to minimize exotic possibilities, for the interest of an infinite lottery as a probe increases the closer its world is to one like ours. Section 5 specifies more clearly just what the lottery's "choosing without favor" must mean. Section 6 makes conservative recommendations over which components may be employed as primitives. Section 7 urges that the infinity of the lottery requires that we admit supertasks, lest the range of possibility be too narrowly restricted and it advises caution in their use, since imprudent use can easily lead to internal contradictions.

The remainder of the paper explores various proposals for infinite lotteries. Section 8 rejects a supertask proposal that employs an infinitely accelerated random walk. Section 9 rejects an ingenious proposal by Hansen (2016) that employs a reversed supertask.

The concluding sections 10 and 11 describe my best candidates for an infinite lottery machine. The weaker one is in Section 10. It employs measurement on a quantum system comprised of a superposition of infinitely many basis states. Section 11 extracts an infinite lottery from an infinite system of binary randomizers ("coin tosses"). The last example is notable in that its individual outcomes and even whether it is successful at all are nonmeasurable in the natural background probability measure.

Readers impatient to get the best answer I have to the "How To ..." of the title should jump to these last sections, although they may find their complexities puzzling without the preparation of the earlier sections. 


\section{Simple Machines that Fail}

It may seem that all this is much ado about very little. Might we not find a simple way to implement an infinite lottery? The trouble is that simple proposals have a maddening habit of falling apart on inspection. Here are three to give a flavor of the problems.

\subsection{The Infinitely Large Urn}

A traditional lottery machine consists of a finite set of identically constituted, numbered balls in an urn. The balls are mixed by tumbling the urn or agitating the contents with stirrers or air jets. One or more balls are then drawn from a recovery point to yield the random outcome. An infinite lottery machine is constituted in the same way, but with infinitely many balls.

We have no assurance that it will work. There are two ways to see the problems. First, informally, the dynamics of mixing is qualitatively different in a finite and an infinite urn. No matter how large the finite urn, if it is tumbled through many revolutions, we have a reasonable expectation that every ball has passed back and forth many times and is thus as likely as any other to be in the vicinity of the recovery point. Infinitely many balls of equal size require an urn of infinite volume. If either tumbled or agitated, how do we know that the result is not merely some local rearrangement of the balls? Thorough mixing will require unlimited speeds so that even the most distant balls can cover arbitrarily large distances in a bounded time to reach the recovery point. How do we realize these speeds throughout the urn? How do we realize them so that each ball has an equal chance of selection?

Second, there are technical results that fail in the infinite case. In statistical physics, a

finite system of hard spheres colliding in a box is a strong candidate for an "ergodic system," that is, loosely speaking, one that over time visits all possible configurations, near enough. That makes it an excellent randomizing device. A lottery machine consisting of finitely many balls in an urn is close enough to it for us to expect comparable behavior. Once we move to an infinite system, however, the technical apparatus breaks down. We no longer have ergodic expectations. Indeed we can no longer even expect deterministic time development.

This last breakdown may seem good. However it is not. For the best we can say is that we just do not know how the infinite system will behave. We need more. We need positive affirmation that the infinite system will behave well as a randomizer. We do not have it. 


\subsection{The Jumping Flea}

The trouble with the infinite urn is that we are unsure of its dynamics. This problem is avoided in another approach that starts with a finite scheme whose dynamics are more clearly defined and takes a limit to infinity. We can choose without favor among $N$ numbers, $1,2,3, \ldots$, $N$ as follows. A flea jumps in one direction only along a long tape with cells marked $1,2,3, \ldots$, $N$, starting at cell 1 . See Figure 1.

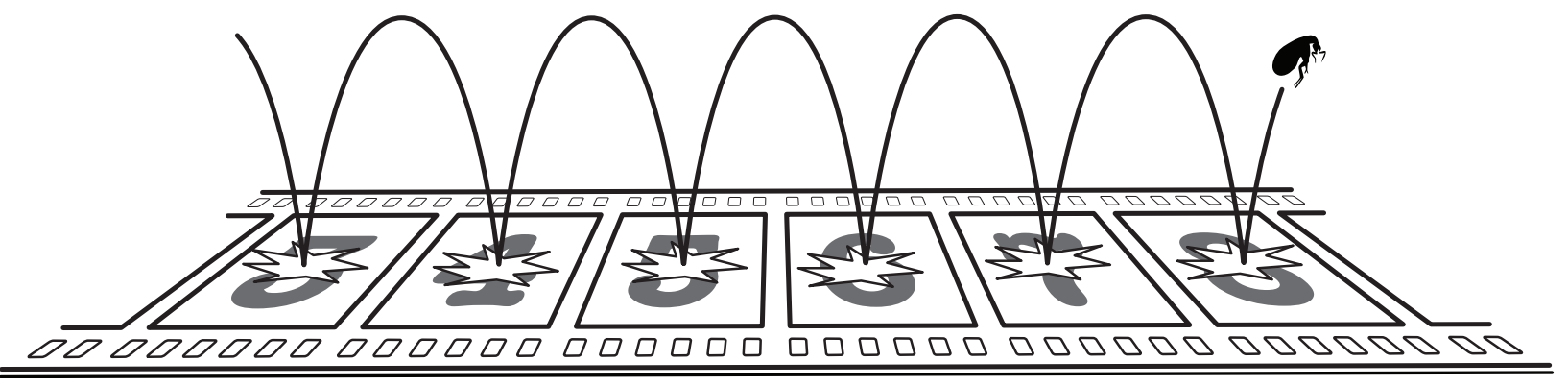

Figure 1. The Jumping Flea

The flea jumps to the next cell only if a randomizer instructs "jump" as opposed to "halt." The probability $P(n, n+1)$ that the randomizer instructs a jump from $n$ to $n+1$ is:

$$
P(n, n+1)=\frac{N-n}{N-(n-1)} \quad \text { for } 0<n<N
$$

A little algebra shows that the flea halts in any of the cells $1,2,3, \ldots, N$ with equal probability $1 / N$.

We can take the limit as $N$ goes to infinity in an attempt to extend this uniform probability to the infinite case. Our hope is that eventually, after some finite but unbounded time the flea will halt on a cell whose number is the outcome. The effort fails. The transition probability (3) becomes, for all $n>0$,

$$
P(n, n+1)=\frac{\infty-n}{\infty-(n-1)}=1
$$


That is, the flea vacates each cell with unit probability, so that, if the limit is taken, the flea has come to rest in no cell. ${ }^{3}$

\subsection{A Spin of a Pointer on Dial}

The last proposal fails since the probability distribution (3) does not behave well in the infinite case. We may try to remedy this deficiency by picking a stochastic system whose probability density is already well-adapted to the infinite case. A uniform probability density over infinitely many outcomes is realized by spinning a pointer over a circular dial with an angular scale etched around its circumference. The pointer is perfectly machined so that no angular orientation is favored and the result is read as an angular position in $0^{\circ}$ and $360^{\circ}$ inclusive, as indicated by the pointer, when it comes to rest. See Figure 2.

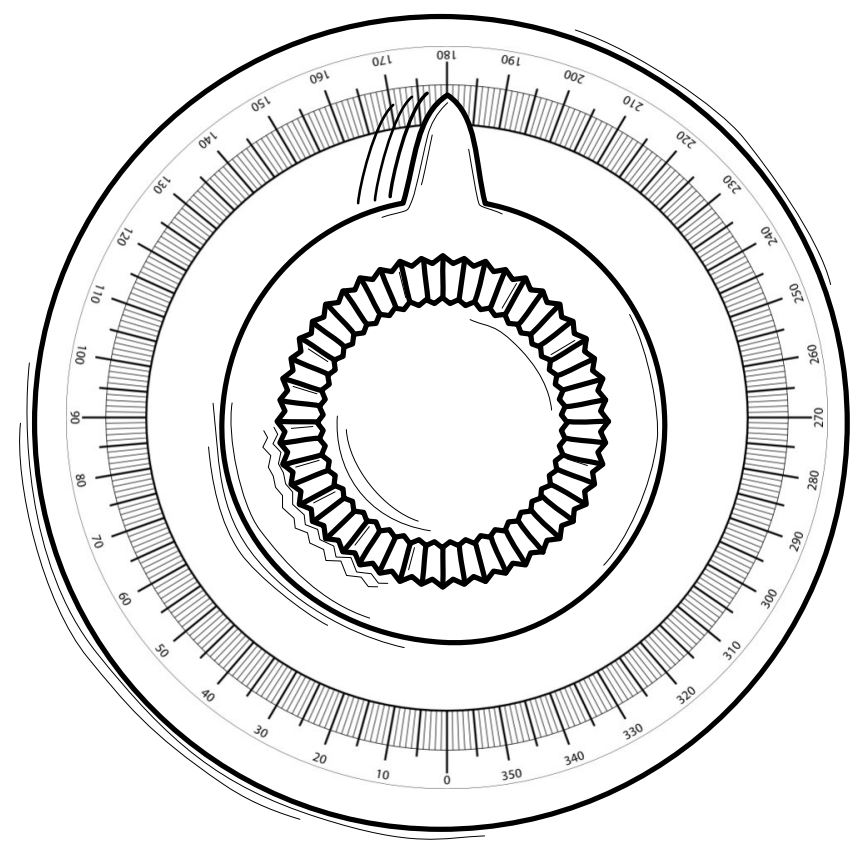

Figure 2. A Spinning Pointer and Dial

${ }^{3}$ If we accelerate the jumping in a supertask, so infinitely many jumps are completed in finite time, the flea will have jumped "off to infinity," behaving like what is called a "space evader" in Manchak and Roberts (2016, §1.4). 
So far, the device chooses randomly among too many outcomes: all of the continuum many, real angles in $0^{\circ}$ and $360^{\circ}$. The infinite lottery must choose among only countably many outcomes, since they can be mapped one-one to the naturals $1,2,3, \ldots$ We adapt the device to this case by selecting a uniformly distributed subset of countably infinite angles: the rational angles. ${ }^{4} \mathrm{We}$ spin the pointer until eventually a rational angle is chosen. It is our outcome.

There are two problems. First, the outcome of a rational angle is a measure zero event in the uniform measure. That means that it occurs with probability zero; and the probability of success remains zero among any finite number of repetitions. For all practical purposes, it will not happen, no matter how often we spin.

Second, we have no way to read the result. The rational angles are densely distributed among the real angles. That means that infinitely many other rational candidates occupy any neighborhood around the outcome, no matter how small the neighborhood. No finite magnification will be sufficient to discriminate these nearby rational angles from the correct outcome. We might attempt a rescue by choosing a discretely spaced set of countable angles, such as $\left\{359^{\circ}, 358^{\circ}, 357^{\circ}, \ldots, 2^{\circ}, 1^{\circ}, 1 / 2^{\circ}, 1 / 3^{\circ}, 1 / 4^{\circ}, \ldots\right\}$. While any outcome in this set can be discriminated from its neighbors with a magnifier of finite magnification, we have lost the assurance that the outcomes are chosen without favor. They are no longer uniformly distributed over the reals.

\section{Against the Possibility of an Infinite Lottery Machine}

The repeated failure of obvious proposals for infinite lottery machines suggests that the problem may be deeper than a mere failure of our powers of imagination. There may be principled reasons behind the failures. Here are two arguments that purport to establish the impossibility of an infinite lottery machine. I explain why both fail.

\footnotetext{
4 They are uniform in the sense that any two angular segments of the same size host rational angles that can be mapped one-one onto each other; and the entire set of rational angles is mapped back to itself if we shift all the angles by adding any fixed rational angle to them.
} 


\subsection{The Argument from Probability}

Spielman (1977, pp. 254-55) admits that he has "not the slightest idea" for how such a random selection could be made. He illustrates the difficulty with the failure of a simple probabilistic scheme that selects a number by translating sequential coin tosses into a binary number. That is, the outcome of sequential coin tosses heads-heads-tails-heads... is recoded as the binary number $1101 \ldots$ For a natural number to be selected, the tosses must terminate finitely. The mechanism that assures this is another sequence of coin tosses, each of which comes after each toss of the original sequence. We terminate when this second series of tosses shows heads for the first time. The probability that the binary number chosen has $n$ or fewer binary digits is just $1-1 / 2^{n}$. Hence with probability $1-1 / 2^{n}$, the number chosen is less than or equal to $2^{n}$. Thus we are near certain that the number will be less than, say, $2^{100}$, even though there are infinitely many more possible numbers.

This scheme favors smaller numbers. Experimenting with other examples like this, one finds quickly that any ordinary probabilistic mechanism ends up probabilistically privileging smaller numbers; and that may seem inevitable when we recall that a probability distribution must normalize to unity. It looks like we are trying to use physics to overturn a mathematical fact, rather like the doomed attempts to square the circle and trisect the angle. The thought can be captured in the following argument:

\section{Argument from Probability}

No probability distribution can jointly satisfy the normalization condition (1) and the equality of probabilities (2). (mathematical fact)

Therefore, no physical machine can realize the distribution. (physical fact)

The weakness of the argument from probability is the first mathematical fact. It is true as long as we consider real-valued, countably additive probability measures. The summation in the normalization condition (1) is undefined as a probability for either of a real, merely finitely additive probability distribution or one with non-standard infinitesimal values. If the probability distribution is one of these, the still true mathematical fact of the argument fails to preclude successful operation of the infinite lottery machine. That is, the inference is a fallacy. 


\subsection{Argument from Two Infinite Lottery Machines ${ }^{5}$}

Consider two lotteries. For any outcome on the first, there are only finitely many smaller numbered outcomes on the second, but infinitely many larger numbered outcomes. Therefore the outcome of the second has, with overwhelming probability, the greater number. The same inference, starting with the second lottery machine, concludes that, with overwhelming probability, the outcome on the first has the greater number. Both cannot be true. Therefore an infinite lottery machine is impossible.

The fallacy of this argument lies in set theory, prior to consideration of probabilities. Consider all pairs of natural numbers $\langle m, n>$. For any particular value of $m$, say $M$, there are infinitely many $n>M$ but only finitely many $n<M$. It does not follow from this that, for all pairs $<m, n>$, there are infinitely many pairs with $n>m$ and only finitely many with $n<m$. The inference from "for any particular value of $m$ " to "for all pairs $\langle m, n>$ " requires us to form the union of the sets $\{n: n<M\}$. While this set is finite for any particular $M$, the union for all $M$ is infinite.

This problem has been posed as a problem for a countably additive probability measure to motivate the restriction to merely finitely additive measures by, for example, de Finetti (1972, §5.6) and Kadane, Schervish and Seidenfeld (1986, §II). Jaynes (2003, §15.3) has challenged the cogency of this motivation.

\section{Physical Possibility}

There is, it seems, no principled barrier to the physical possibility of an infinite lottery machine, yet ordinary proposals fail reliably. If an infinite lottery is possible, we will need to entertain more exotic proposals. The goal is to use the infinite lottery machine as a foil for testing the reach of inductive inference. Hence we can entertain more exotic proposals, as long it is reasonable to expect some extended form of inductive inference, perhaps non-probabilistic, to apply in the more exotic domains. For such domains, it is sufficient ${ }^{6}$ that the domain:

\footnotetext{
5 I learned of this argument from the ever-inventive Jim Brown.

6 These conditions may not be necessary. Paraconsistent logics support deductive reasoning in domains with inconsistent descriptions.
} 
(a) can be described without logical contradiction (hence non-trivial deductive inference is possible) and;

(b) is governed by some regularities (so inductive inference is possible).

How exotic can a proposal be before it can no longer be judged admissible? In a whimsical piece that conveys skepticism through the woes of label independence of Section 5 below, McCall and Armstrong (1989, p. 223) imagine a lottery constituted as: "In an entirely fair and purely random way, God would simply choose a number." Absent some precise specification of the powers of a supernatural being, we have no assurance that the flight to supernatural powers does not hide logical contradiction or miraculous violation of the domain's regularities.

The sections following delineate the sorts of possibilities that would admit an infinite lottery machine and do so in a conservative way, in order to minimize departures from the actual world.

\section{What Choosing Randomly without Favor Means}

Choosing randomly without favor cannot merely mean choosing with equal probability, for the notion of probability may not apply everywhere in these more exotic domains. In its place, I will revert to a more generic notion of "chance," where this notion should not be understood as a synonym for an additive probability measure. It just designates the indefiniteness of outcomes in a process whose outcome is not determined by the initial set up. The notion of label independence captures what it is for a chancy machine to operate without favoring any of the outcomes.

The notion is recoverable from familiar, finite randomizers, such as dice. They treat each possible outcome with equal favor because of their physical constitutions and their dynamical operations. A fair die is a perfect cube of uniform density in which all faces are equivalent and it must be tossed so that it tumbles freely, eliminating any bias in the die's initial orientation.

This characteristic can be represented formally as label independence. Each face of the die carries a unique label, as "1," “2,” ..., “6.” Its proper physical functioning is unaffected if we peel off, switch around and reattach the labels arbitrarily. That is, we permute the labels

arbitrarily, where a permutation is a one-one mapping of the label set back onto itself, and require: 


\section{Label independence}

All true statements pertinent to the chances of different outcomes remain true when the labels are arbitrarily permuted.

For example, true, pertinent statements concerning a fair die are:

Faces 1 and 3 are the same size, shape and weight.

One of faces 1,3 or 5 come up roughly three times as often as face 2 in repeated throws.

If we permute the numbers according to $1 \rightarrow 2,2 \rightarrow 1,3 \rightarrow 4,4 \rightarrow 3,5 \rightarrow 6,6 \rightarrow 5$, then these true statements become true statements:

Faces 2 and 4 are the same size, shape and weight.

One of faces 2, 4 and 6 come up roughly three times as often as face 1 in repeated throws.

The phrase "pertinent to the chances" is essential, for not all truths are preserved by label permutation. For example, almost universally, dice are made so that the numbers on opposite faces sum to seven, although this design feature is not pertinent to their randomizing function. Consider the statement, likely true of any die you may own:

The 1 and the 6 are on opposite faces

It does not remain true under permutation of the labels. However this failure does not compromise label invariance since the statement is not pertinent to the chances. As these examples show, whether something is pertinent to the chances is decided by the particular physics that governs operation of the randomizer.

Label independence is a necessary property of any randomizer that selects among outcomes without favor. Therefore we must require it for an infinite lottery machine. This requirement has the immediate consequence that even a finitely additive probability measure is not the appropriate conception of chance for the infinite lottery machine. This failure results from permutations of infinite sets in which an infinite set whose complement is infinite can be mapped onto either of two of its proper, disjoint, infinite subsets. Hence, by label independence, 
the original set must have the same probability as each of these disjoint subsets, which violates additivity, unless the sets have probability zero. ${ }^{7}$

Bartha $(2005, \S 5)$ and Wenmackers and Horsten (2013, p. 41) both give up label independence, finding the abandoning of an additive probability measure too great a sacrifice. In my view, label independence cannot be abandoned since it is a consequence of the defining characteristic of an infinite lottery: it chooses its outcomes without favoring any of them. If we require that an additive measure can describe the chance properties of the infinite lottery machine without contradiction, we have replaced the original requirement of selection without favor, by selection without favor under some added restriction, such as requiring the selection to respect a preferred numbering scheme.

In the following, label independence of any proposal for an infinite lottery machine will be required. Given the tension between probability measures and a label independent infinite lottery machine, we might expect outcomes that are not measurable according to whichever background probability measure is available. This expectation is realized in the proposal of Section 11 below.

\section{Which Components May an Infinite Lottery Machine Employ?}

A proposal for an infinite lottery machine must draw on some inventory of devices and processes that we deem physically admissible. The difficulty is that there are very few true randomizers in our physics. 8 Prior to the early twentieth century, physical processes were assumed to be deterministic. The paradigmatic randomizers, coin tosses, die throws, card shuffles, roulette wheels and the like, were assumed deterministic. The appearance of randomness in real coin tosses was assumed to arise from the system's high sensitivity to initial

\footnotetext{
7 The difficulty is well-known developed and developed more precisely in Bartha $(2004, \S 5)$ and Norton (2011, pp. 412-15).

8 To keep the selection conservative, I set aside Newtonian indeterministic systems such as the dome or the infinite of masses-spring-mass-spring-... system, described in (Norton, 2008: 1999, $\S 1.1$ ), even though van Strien (2014) has shown that dome-like indeterminism had alreadybeen found in Newtonian physics in the nineteenth century.
} 
conditions and our practical inability to control these initial conditions closely enough to produce predictable outcomes.

Serious consideration of indeterministic systems entered the mainstream of physics only with quantum mechanics. Even then, it arose in just one, ever-troublesome process: quantum measurement, also known as the "collapse of the wave packet." Otherwise the time evolution of

quantum systems respects determinism more than systems of many other physical theories. Some accounts of quantum theory, such as the Bohm or the Everett account, even dispute whether the quantum measurement is truly indeterministic.

Given these complications, the appropriate way to proceed is to admit as primitive components for an infinite lottery machine whichever randomizers are commonly treated in the literature as unproblematic. Otherwise we encumber ourselves with problems that are not peculiar to the specific task of designing an infinite lottery machine. These randomizers are of two types: devices like tossed coins and thrown dice that select with equal probability among a finite number of outcomes, where that number is usually small; and quantum systems that collapse on measurement onto one of a set of basis states associated with some measurement operator.

We shall strain below to find a scheme that conforms with these restrictions. They may well start to look adverse and even perverse. Why expect that an infinite lottery machine can be constructed from finite randomizers? Why not simply posit at the outset an infinite lottery machine as an idealized possibility to which our theories of chance must be responsible? We did just that for centuries with finite randomizers. A coin toss and a die throw were accepted as true randomizers for the sake of probabilistic theorizing, even though prior to quantum theory the dominant understanding of physical theory was that both are deterministic systems.

\section{The Limitations of Finitude: When Supertasks Can Help}

\subsection{When Supertasks are Needed}

An infinite lottery machine employs an infinite set of outcome states that are, commonly, discretely arranged. Ordinary processes that complete only finitely many distinct actions may not be able fully to operate these infinite machines. Their operation may need completion of 
infinitely many distinct actions. If this infinity of actions is completed sequentially, starting with the first, in a finite time, they must be accelerated arbitrarily quickly. If the process has sequentially actions $\mathrm{A}, \mathrm{B}, \mathrm{C}, \mathrm{D}, \ldots$, they might each need time $1 / 2,1 / 4,1 / 8,1 / 16, \ldots$ for completion. Then a total of $1,2,3,4, \ldots$ actions are completed at total elapsed times $1 / 2,3 / 4$, $7 / 8,15 / 16, \ldots$ so that all are completed by time 1 . What results is a supertask. 9

The simplest, benign example is Zeno's dichotomy. To complete the race, a runner runs half way to goal (action A), then half way again (B); and then half way again (C); and so on infinitely, completing each stage at times $1 / 2,3 / 4,7 / 8, \ldots$

Supertasks are needed when a finite randomizer is used repeatedly, sequentially in time, in the operation of the infinite lottery machine. If the ill-fated "jumping flea" is to have the possibility of accessing all infinity of the cells on the tape, its random jumps must be accelerated so that arbitrarily many could be completed in some fixed, finite time.

One might imagine that the awkwardness of a supertask can be avoided by operating the randomizers in parallel in time. That will require an infinity of randomizers, such as needed in the scheme in Section 11 below. This escape only postpones the trouble since the infinity of randomizers will occupy an infinite volume of space. We now need a process that can survey the state of the infinite randomizer system, recover the outcome and communicate it to the observer's location in space, in a reduced form intelligible to an observer with finite capacities. A surveying and communication process that operates at a fixed speed will not suffice. For any fixed speed, we can always find distances sufficiently great that arbitrarily great times will be needed for the survey. The problem can be solved by a supertask. We employ a process whose parts run at successively greater speeds, so that, for any arbitrarily large distance, the time needed for survey and communication over it can be kept below some fixed bound.

One might again imagine a further escape that selectively shrinks the infinite set of randomizers so that infinitely many will fit into a finite space. ${ }^{10}$ This escape once again merely postpones the problem. To read the outcome, we finite observers require indefinitely fine powers

\footnotetext{
${ }^{9}$ For an introduction to the extensive literature on supertasks, see Manchak and Roberts (2016).

10 Davies (2001) describes a supertask computer able to compute Turing non-computable functions through its use of unlimited miniaturization.
} 
of discrimination to discern the states of arbitrarily small system, arbitrarily close to their neighbors.

We may be able to acquire these powers through a supertask. How this works can be seen in the simpler problem of discerning the reading of a pointer on a finite scale that shows markings $1,2,3, \ldots$ We might imagine it as delivering the infinite lottery machine's outcome. In a favorable case, the markings are discretely spaced and well-ordered in space, as shown in Figure 3. They still must approach at least one accumulation point and most outcomes will reside within arbitrarily small neighborhoods of the accumulation points.

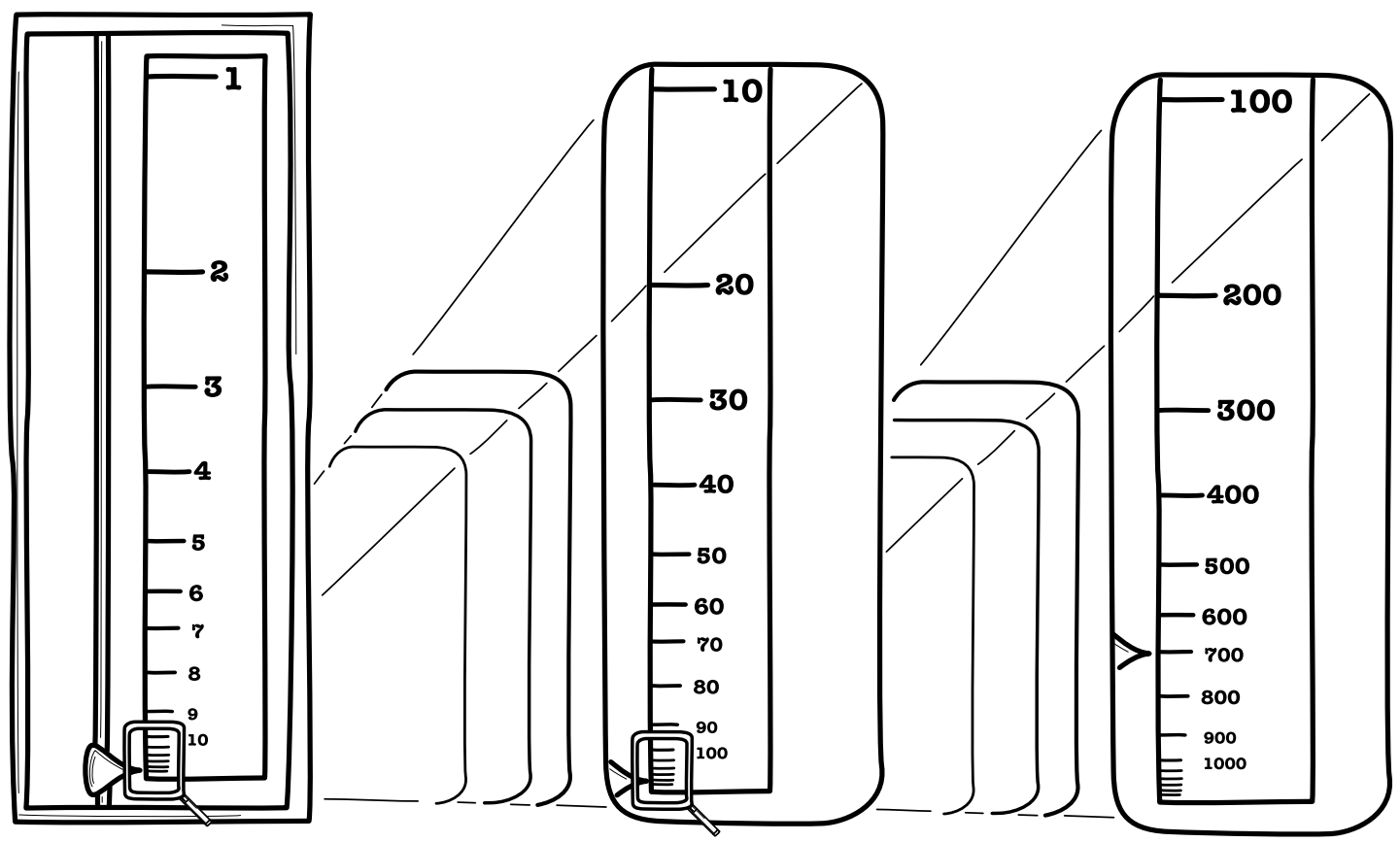

Figure 3. Pointer on a scale with Infinitely Many Markings

In the non-atomic, continuum world in which such a pointer-scale combination is possible, one might think that we could read the outcome simply by inspecting the scale with an “infinitely powerful” magnifier. However such a device would not produce an image of the scale for us to inspect, for it would infinitely separate any two points on the scale, no matter how close. It would at best tell us whether the single point on the scale at which it is focused is the outcome or not. Thus it could not be used to learn the outcome, but only to confirm that some nominated value is the outcome. A scheme that can enable us to learn the outcome is a magnifier, whose magnification could be increased arbitrarily. We would then inspect the scale 
sequentially using magnifications $10 x, 100 x, 1000 x, \ldots$ as shown in Figure 3, until sufficient, finite magnification is achieved to enable the result to be discerned. Since arbitrarily high magnification is needed to be assured of a successful reading, the sequential inspections would need to be carried out on an accelerating schedule as a supertask, so that arbitrarily many of them can be completed within some fixed time limit.

If the scale markings corresponding to the different outcomes are not discretely spaced but are dense, as in Section 2.3, then not even this supertask will enable the outcome to be read.

The example of the infinitely large urn of Section 2.1 shows how intimately related these problems are. If we have infinitely many, equal sized balls, how do we mark them so we can read the number of the ball selected? We must somehow fit the arbitrarily many digits needed to represent arbitrarily large numbers onto the finite area of a ball. That can only be done if the digits are made arbitrarily small. Once again we would need indefinitely fine powers of discrimination to read the outcome. Or if we encode the outcome in colors, we need the power to discriminate arbitrarily close colors.

Supertasks bring dangers unfamiliar to their finite brethren. We shall consider two types: ordinary and reversed. Each has its unique problems.

\subsection{The Dangers: Ordinary Supertasks}

An ordinary supertask accelerates forwards in time. A simple example illustrates their dangers: their end states may not be well-defined. ${ }^{11}$ Two trains approach, while a bird flies rapidly to and fro between their fronts along a straight line besides the tracks, as shown in Figure 4. The bird starts one hour ahead of the meeting in line with the leftmost train. By the time the trains pass, the bird will have reversed direction, left-right-left-right-... infinitely many times and completed a supertask. At the moment the fronts of the trains pass, the position along the tracks of the bird is well-defined: it is just in line with the position at which the fronts of the trains pass. In which direction is the bird facing at this moment? The bird has completed the infinite sequence left-right-left-right-... and, we might expect naively that the direction of travel is just

11 This supertask has a venerable history that includes a celebrated von Neumann anecdote. See Halmos (1973, pp. 386-87). 
the "last member" of this infinite sequence. Of course there is no last member and no limit to which the sequence converges. The direction is undefined.

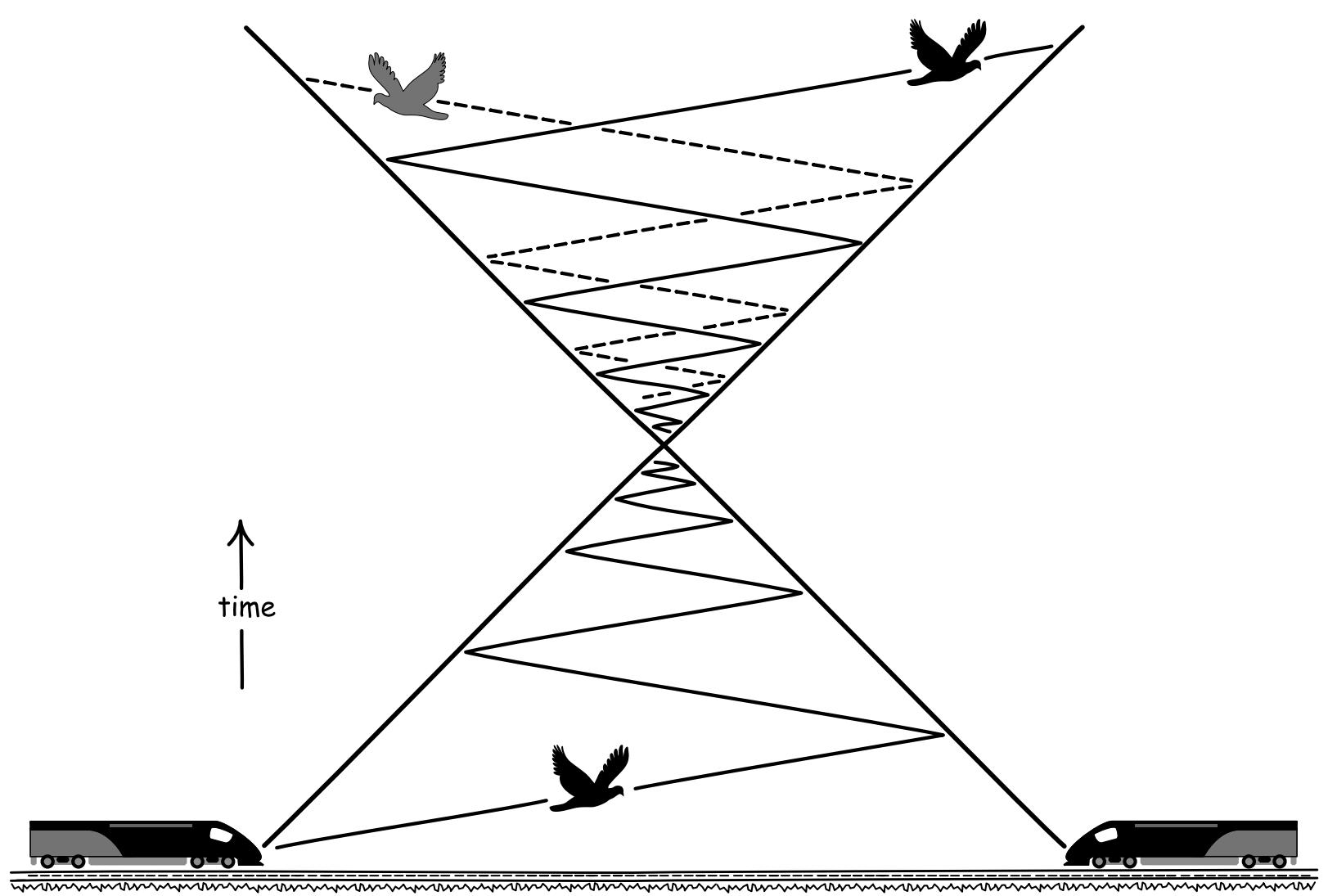

Figure 4 The Trains and Bird Supertask and Reversed Supertask

The moral to be applied below is that supertasks can have well-defined end states. The position of the bird is well-defined at the moment the trains pass. But they also may not have well-defined end states, as shown by the direction of flight at that moment.

\subsection{Reversed Supertasks}

Reversed supertasks proceed as does an ordinary supertask, but reversed in time. One arises if the bird of the last supertask keeps flying after the trains have passed one another. In order to arrive at any future position, the bird must have completed an infinity of direction changes in a manner akin to the temporal inverse of the direction changes of the original supertask, as shown in Figure 4. 
It is easy to imagine that the trajectory of the bird in the reversed supertask is fixed by its past motion. By symmetry in time, we may argue, the bird merely traces the earlier trajectory, reflected left-to-right, in reversed temporal order. An hour after the trains passed, the bird is inline with the front of the rightmost train. However that is not so. Infinitely many future motions are possible. The easy proof is to assign the bird at any position between the trains, flying in either direction, one hour after they passed. Whichever is chosen, if we now project the motion of the bird backwards, as shown by the dashed trajectory in Figure 4, we find that it will oscillate to and fro infinitely often along a path that starts at the moment and position at which the trains pass.

Bernadete's (1964, pp. 259-60) venerable reversed supertask describes a walker who plans to go from A to B. An infinity of gods is arrayed along the path at the $1 / 2,1 / 4,1 / 8, \ldots$ mile marks, each determined to throw up a wall to block the walker, unless another god has earlier done so. No god needs to throw up a wall at its location, since the walker would have been blocked by the wall of an earlier god. We are to conclude, then, that the walker is halted at the outset by no god at all, but merely by their intentions to act that somehow creates a mysterious stopping force.

That conclusion should be resisted. No conclusion is possible unless we specify the dynamical laws governing the motion of the walker. The obvious law is that the walker proceeds if and only if the walker is not impeded by a wall. With that law, the only conclusion that we can come to is that the initial set up admits no future for the walker consistent with all its assumptions.

The moral to be applied below is that reversed supertasks are especially delicate. The familiar pathologies of infinite limits may mean that initial conditions can fail to determine uniquely the future course of the supertask (bird-trains example); and that there may even be no future behavior at all consistent with the specification (walker-gods example).

\section{The Infinitely Accelerated Random Walk}

A walker steps back and forth along an infinite road, proceeding over a countable infinity of cells marked $x=\ldots,-2,-1,0,1,2, \ldots$ The walker starts at cell $x=0$ at stage $t=0$. With each 
step to stages $t=1,2,3, \ldots$, the walker moves up one (“+ +1 ”), down one (“-1”) or stays put (“0”), with probabilities:

$$
P(+1)=P(-1)=1 / 4 \quad P(0)=1 / 2
$$

The probability that the walker is in cell $x= \pm n$ for $\mathrm{n} \geq 0$ at stage $t=N$ is ${ }^{12}$

$$
P(n, N)=P(-n, N)=\frac{(2 N) !}{(N+n) !(N-n) !} \frac{1}{4^{N}}
$$

where the walker is confined to cells $-N \leq n \leq N$. The probability distribution adopts a bell-like shape, as shown in Figure 5.

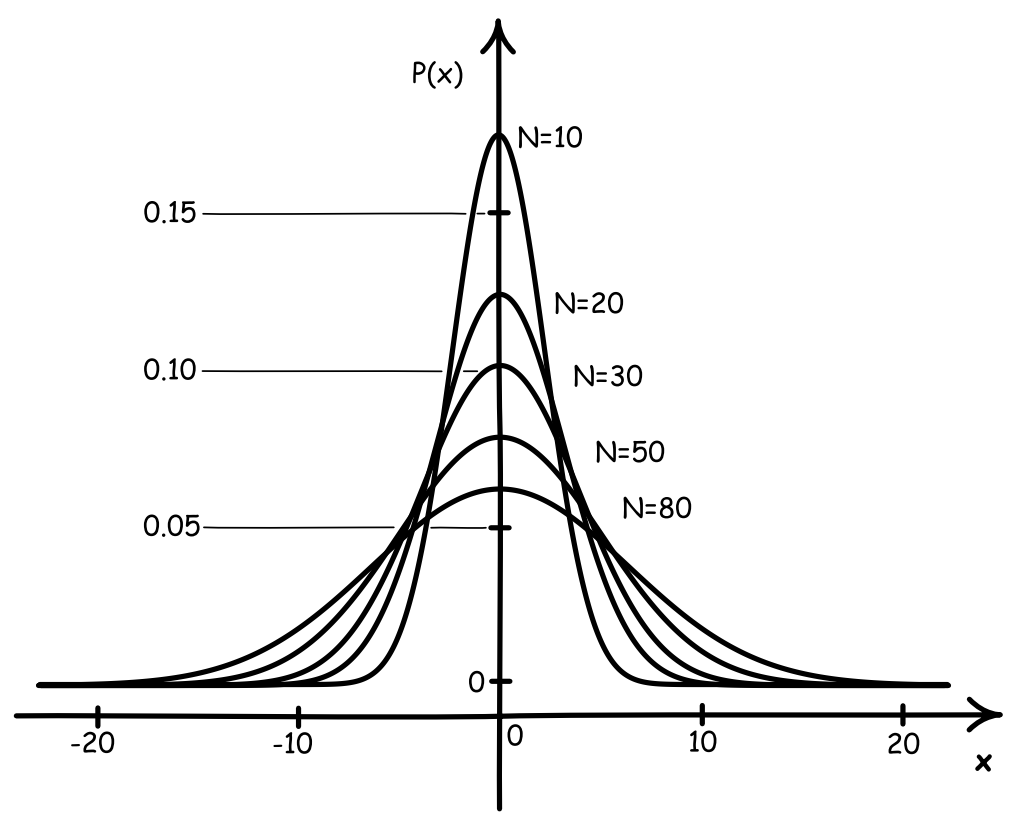

Figure 5 Position Probability Distributions for Different Stages N

12 The fastest way to arrive at (5) is to generate the random walk from a simplified half-step random walk, in which the walker can only move up a half step (“+1/2") or down a half-step (“-1/2”) with probability $1 / 2$ in each case. The result of two half steps is $+1,-1$ or 0 , conforming with the probabilities of (4). In $N$ steps $=2 N$ half-steps, the probability of $m$ half steps up and $2 N-m$ half steps down is just $\frac{(2 N) !}{m !(2 N-m) !} \frac{1}{2^{2 N}}$. This results in a net motion of $m-(2 N-m)=2(m-$ $N)$ half steps up, which corresponds to the walker moving to cell $n=(m-N)$. This last probability becomes equation (5) with the substitutions $m=N+n$ and $2 N-m=2 N-(n+N)=N-n$. 
As the number of stages increases, the walker meanders over greater distances and the probability distribution flattens. ${ }^{13}$ It approaches arbitrarily closely to the limit curve $P(n, \infty)=0$, which is uniform over all the cells $n$. This limit is the sort of distribution we expect for an infinite lottery. It respects label independence since the uniform limit treats each cell alike, independent of its label.

Does this mean that we can realize the infinite lottery machine merely by accelerating the motion of the random walk through a supertask, in which infinitely many stages are completed in finite time? Alas, no. The infinite lottery machine will fail to return a definite outcome through a failure of convergence, analogous to the failure of the bird in the ordinary supertask of Section 7.2 to converge to a definite direction at the completion of its supertask.

At any finite stage $\mathrm{N}$ of the supertask, the walker will occupy a definite cell number. The set of accessible cells is the outcome space for the probability distribution (5), which measures our uncertainty over which cell is occupied. The corresponding outcome space associated with the completed supertask is the set of cell numbers to which the walker's position converges in the infinite limit. The trouble is that there is no such limit, other than in exceptional, measure zero cases. For example, if the walker's true motions were $+1,0,-1,0,+1,0,-1,0,+1,0,-1,0$, $\ldots$, then the walker would oscillate indefinitely over cells $x=0$ and $x=1$, without convergence. One might imagine that the probability, in some extended sense, at the completion of the supertask is spread over the cells through which these oscillations had moved endlessly. But whatever such an extended probability measure may mean, there is no definite outcome behind it in the form of a single occupied cell. Thus this infinite lottery machine fails to deliver a definite outcome.

13 That is, for any $\mathrm{m}, \mathrm{n}, \lim _{N \rightarrow \infty} \frac{P(m, N)}{P(n, N)}=\frac{(N+n) !(N-n) !}{(N+m) !(N-m) !}=1$. 


\section{Hansen's Reversed Supertask Infinite Lottery Machine}

Hansen (2016) has devised an ingenious reversed supertask as a candidate infinite lottery machine. An urn holds an infinity of balls numbered $1,2,3, \ldots{ }^{14}$ An infinity of gods act according to the following instructions that form a reversed supertask, with an infinity of actions taken at times $t=\ldots, 1 / n, \ldots 1 / 4,1 / 3,1 / 2$.

At time $t=1 / \mathrm{n}, \operatorname{god}_{n}$ receives the urn with exactly $n+1$ balls and randomly chooses to discard one, passing the resulting urn with $\mathrm{n}$ balls to $\operatorname{god}_{n-1}$.

At time $t=1 /(n-1), \operatorname{god}_{n-1}$ receives the urn with exactly $n$ balls and randomly chooses to discard one, passing the resulting urn with $n-1$ balls to $\operatorname{god}_{n-2}$.

At time $t=1 / 2, \operatorname{god}_{2}$ receives the urn with exactly 2 balls and randomly chooses to discard one, leaving the resulting urn with 1 ball only.

Working backwards through the steps, we see that at all times $t=1 / n$, for $n=2,3,4, \ldots$, there were $n+1$ balls in the urn. The number grows without bound, so that at the outset at $t=0$, there were infinitely many balls in the urn. If the instructions are carried out as described, then the resulting final ball will have been chosen from that infinity without favor, as the lottery requires. The supertask respects label independence.

Both Hansen and Kerkvliet (2016) argue that the reversed supertask fails; and I agree with them. We each give slightly different diagnoses. In my view the decisive problem is just the problem that we saw with the reversed bird-train supertask of Section 7.3: the initial conditions fail to specify uniquely what will transpire. The nature of the indeterminism varies according to the default rule for each $\operatorname{god}_{n}$ if the god is not given an urn with exactly $(n+1)$ balls. If the default rule is that the god then just passes on the urn as it was received, then the initial conditions are compatible with two time developments: the one intended and one in which no balls are ever removed. Or if the default rule is that the god then just removes any single ball, if there are any

14 To avoid issues of failure of convergence in the positions of the balls in the past time limit, it is convenient to replace the urn and balls with an infinite panel of numbered switches. Removing a ball corresponds to setting its switch from "in" to "out." 
to remove, then the final state of the urn can have a set of balls of any size: zero, any finite number or any infinite number whose complement set is also infinite.

Hansen seeks to block this indeterminism by the default rule, here called "all":

If the $\operatorname{god}_{n}$ is not given an urn with exactly $(n+1)$ balls, it passes on the urn

containing just all $\mathrm{n}$ balls numbered $1,2, \ldots, n$.

This rule, it seems, blocks the possibility of any $\operatorname{god}_{n}$ receiving an urn with anything other than $(n+1)$ balls. For that can only happen if $\operatorname{god}_{n+1}$ passes $\operatorname{god}_{n}$ an urn with other than $(n+1)$ balls. That cannot happen since the new default rule would preclude $\operatorname{god}_{n+1}$ doing this; and so on for $\operatorname{god}_{n+2}, \operatorname{god}_{n+3}, \ldots$ The curious and crucial consequence is that, reasoning by elimination, this new default rule blocks all but the intended time development, while the default rule is never actually activated. That is, no god actually acts as the default rule requires. That it is never activated is crucial, since its activation would compromise the randomizing process.

The trouble with this default rule is that it is inconsistent with the dynamics presumed for the urn: a rule can only have an effect if it is activated. Yet somehow this unactivated rule manages to block many possible time developments. The fallacy lies in reasoning by elimination. It is cogent only if we know that an occurring physical process eliminates the unwanted outcomes that would otherwise happen. In this case there is no such process occuring.

The simplest way to see the problem is to consider a neighboring default rule that we shall call "even":

If the $\operatorname{god}_{n}$ is not given an urn with exactly $(n+1)$ even numbered balls, it passes on the urn containing just the $n$ even numbered balls, $2,4, \ldots, 2 n$.

Reasoning by elimination as before, we conclude that each god receives an urn containing only even numbered balls, merely in virtue of the presence of the unactivated rule "even." Since each $\operatorname{god}_{n}$ only ever receives an urn with even numbered balls, none of the gods removes an odd numbered ball from the urn. Yet there are no odd numbered balls in the urn for all $t>0$. This contradicts the presumption that a ball only disappears from the urn if a god removes it. With these default rules, the supertask now fails in the second way indicated in Section 7.3. The presumed behavior is inconsistent with the dynamics governing the system.

Another way to see the inconsistency is to imagine three versions of the supertask that differ only in the default rule used: "all," "even" and "odd," respectively, where "odd" has the 
natural definition in analogy to "even." In each version, the default rule is never activated. Therefore the default rule can make no difference to the outcome. Yet it does if the eliminative reasoning is respected. In the case of "all," the outcome can be any ball number. In the case of "even," it can only be an even numbered ball. In case of "odd," it can only be an odd numbered ball.

There is a final problem even if the supertask proceeds as intended. It arises if we assume countable additivity for the probability measures used in the ball selection. (In that case, we can consistently assign no probability to the selection of any individual ball.) The probability that one or more of the lowest numbered balls is removed by the god-collective ${ }_{N}=\left\{\operatorname{god}_{N}, \operatorname{god}_{N+1}\right.$, $\left.\ldots, \operatorname{god}_{2 N-1}\right\}$ is one half. 15 The urn passed to $\operatorname{god}_{N-1}$ has already been acted on by infinitely many god-collectives: god-collective ${ }_{N}$, god-collective ${ }_{2 N}$, god-collective gn $_{3 N}, \ldots$ With a probability of one half, each god-collective removed one or more of the lowest numbered balls. Hence with probability one, computed by a limiting process authorized by countable additivity, infinitely many of the lowest numbered balls have been removed by them; and the urn is empty by the time $\operatorname{god}_{N-1}$ receives it; and it is so for any N. Since successful operation of the lottery requires that the urn never be empty, we have, with probability one, that the lottery fails to operate successfully.

\section{A Quantum Mechanical Infinite Lottery Machine}

Quantum mechanics holds great promise for proposals of infinite lottery machines. Its standard interpretation is the one place in which practical, modern science has irreducibly random processes. It turns out, however, that quantum infinite lottery machines of simple design face difficulties similar to those we have seen.

Quantum randomness derives from one process: quantum measurement. We measure a system that is in a superposition of states and in the process, originally known as "collapse of the wave packet," it randomly adopts one of the states in the superposition. The collapse can be

15 The probability that none of the gods in the collective remove the lowest numbered ball from its urn is $\frac{N}{N+1} \times \frac{N+1}{N+2} \times \cdots \times \frac{2 N-1}{2 N}=\frac{N}{2 N}=\frac{1}{2}$. 
effected in any of the degrees of freedom of a quantum system. Real life quantum random number generators most commonly use photons and exploit collapse in particle spin degrees of freedom, in particle arrival times, in particle positions and in particle numbers. For a recent survey, see Ma et al. (2016).

Most of these schemes do not extend especially well to the infinite case. Spin observables adopt finitely many values only. Arrival time and position are each continuous magnitudes, whose outcome must somehow be reduced to a countable infinity. They face the same problems as the spinning pointer machine of Section 2.3.

More promising are systems with infinitely many discrete states. One is the superposition of one-photon, two-photon, three-photon, ... states $|1\rangle,|2\rangle, \mid 3>, \ldots$ in a coherent superposition, such as is produced by a laser:

$$
\left|\alpha>=\mathrm{e}^{-\frac{|\alpha|^{2}}{2}} \sum_{n=0}^{\infty} \frac{\alpha^{n}}{\sqrt{n !}}\right| n>
$$

where $\alpha$ is a complex field amplitude and $\mu=|\alpha|^{2}$ is the mean photon number. When the particle number $n$ is measured, the coherent state collapses to one of the $n$-photon states $\mid n>$, where $n$ is Poisson distributed. Such randomizers are practical and have been built, as described in Applegate et al. (2015).

To adapt this randomizer to an infinite lottery, we replace the normalized superposition (6) by an unnormalizable 16 superposition

$$
|1>+| 2>+\ldots .+\ln >+\ldots .
$$

of n-particle states, $\mid n>$. On measurement of the particle number, this state collapses to one of the infinitely many states $\mid n>$, without favor to any. In this regard, it conforms with label independence.

There are two problems with this scheme. ${ }^{17}$ First is technical: because the state of (7) is unnormalizable, it is a vector in a vector space, but not in a Hilbert space, which is the structure

16 For a concern that quantum theory likely precludes such unnormalizable states, see Earman (manuscript).

17 This scheme can also be implemented (but with the same two problems) as an infinite superposition of the discrete energy eigenstates of a particle in an infinitely deep potential well; or a harmonic oscillator. 
upon which ordinary quantum theory is built. The application of the Born rule for the probability of different outcomes is problematic in precisely the same way as is application of probabilities to the infinite lottery. These problems can be solved by liberalizing the mathematical formalism. For the physical ideas behind quantum theory assure us that state (7) will collapse on measurement to one of its components.

The second problem cannot be solved by such liberalization. The energy of the $n$ particle state increases linearly with $n$. Hence the state (7) is associated with an unbounded energy in the following sense: for any finite energy $E$, there will be only finitely many states in (7) with energy less than $E$, but infinitely many with energy greater than $E$. Unbounded energy makes creation of the state dubious and, if constructed, will destroy any localized measurement device.

We can attempt a solution by exploiting systems with infinitely many discrete states, whose energies are bounded from above and below. A simple case is the infinitely many $\mathrm{s}$ electron orbitals adopted by an excited electron in a stationary state in a hydrogen atom. The energies $E_{n}$ of the $n$ s orbitals for $n=1,2,3, \ldots$ coincide with those of the original Bohr theory of the atom of 1913 (following Sommerfeld, 1923, p. 214):

$$
E_{n}=\frac{-2 \pi^{2} m e^{4}}{h^{2}} \cdot \frac{1}{n^{2}}
$$

for an electron of charge $e$, mass $m$ and Planck's constant $h$. The energies vary from the ground state energy $-\left(2 \pi^{2} m e^{4}\right) / h^{2}$ to approaching arbitrarily close to energy 0 as $n$ grows towards infinity.

For the infinite lottery machine, we prepare an equally weighted superposition of the $n \mathrm{~s}$ orbitals for all $n$, so that each orbital $n$ s corresponds to $\mid n>$ in (7). Upon measurement the superposition collapses to one of the states $\mid n>$ as before.

There are, once again, problems. First, we still have the technical problem of an unnormalized state (7). Second, for large $n$, the energy of the orbital is very close to 0 , which is the energy at which an electron is liberated from the atom. Hence, the slightest increase in energy from, for example, a thermal fluctuation, will eject the electron from the atom. Infinitely many states in the superposition are within any arbitrarily small energy of this zero energy level. Hence the superposition is very unstable. Third, because the energy of infinitely many of the states are clustered so closely together, measuring the energy of the collapsed state will once again require the troublesome capacity to make indefinitely fine discriminations. 
The most direct measurement scheme is to allow the excited electron to drop back to the lowest energy ground state $n=1$ and measure the frequencies of the resulting emitted photon or photons. That measurement will collapse the superposition to one of the infinitely many orbitals undergoing decay. The energy of that orbital is determined by the measured frequencies of the emitted photon or photons. For the simplest case of a single jump to the $n=1$ ground state, a single photon is emitted with the frequency $\mathrm{v}$ :

$$
v=\frac{2 \pi^{2} m e^{4}}{h^{3}} \cdot\left(1-\frac{1}{n^{2}}\right)
$$

For the infinitely many large $\mathrm{n}$ states, the frequency $v$ will be almost exactly $\left(2 \pi^{2} m e^{4}\right) / h^{3}$ and arbitrarily fine discrimination in measurement of $v$ will be needed to separate neighboring values of $n$, as $n$ grows large.

Finally, the size of the electron orbitals grows arbitrarily large. Their Bohr atom radii grow with $n$ as (Sommerfeld, 1923, p. 212): 18

$$
r=n^{2} \frac{h^{2}}{4 \pi^{2} m e^{2}}
$$

So the superposition requires that the universe be empty of other atoms, for otherwise Coulomb repulsion from these other atoms' electrons would confine the original atom's electron and alter the energy relation (8), so that the electron energy is no longer bounded from above.

\section{The Infinite Array}

An infinite lottery machine requires exotic processes. The best will employ the minimum of them; and this section will describe my best proposal for such a minimum. In the proposal, the processes of selection of the number are separated from those that communicate the number to us. The processes of selection require no supertasks and, with just a little indulgence, can be mapped onto real systems in our universe.

18 A more modern calculation (Johnson, 2007, p. 34), using the Schrödinger wave for the electron orbitals, affirms that the position expectation for the $n$th s state $<r>$ grows with $n^{2}$. 


\subsection{The Selection of the Number}

The system consists of an infinite, two-dimensional array of independent, two-state randomizers. For simplicity, imagine each randomizer as a coin toss with equal probabilities of heads $\mathrm{H}$ and tails $\mathrm{T}$ :

$$
\mathrm{P}(\mathrm{H})=\mathrm{P}(\mathrm{T})=1 / 2 .
$$

The coins are all flipped at the same moment.

Our interest is a countable subset of the row configurations that encode the natural numbers $1,2,3, \ldots$ A simple coding system encodes the number $\mathrm{n}$ as a row whose first $\mathrm{n}$ terms are $\mathrm{H}$ and all remaining terms are $\mathrm{T}$. The number 5 is encoded uniquely as HHHHHTTTTTTTTTTTTT... The outcome of the infinite lottery machine is the natural number encoded in the array in the first row that successfully encodes a natural number, if there is one, as shown in the marked row in Figure 6.

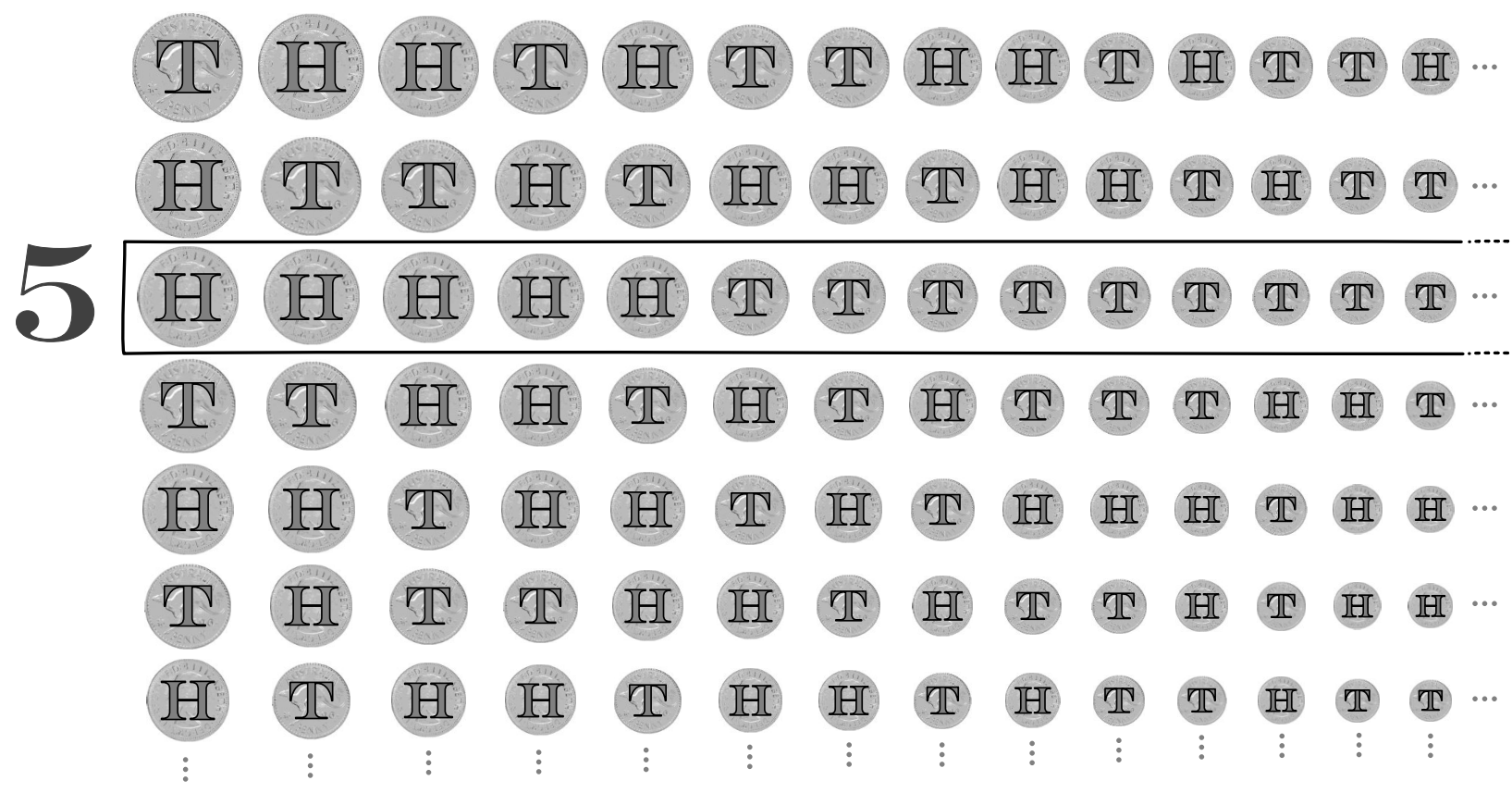

Figure 6. An Infinite Array of Binary Randomizers

\subsection{Label Independence}

Label invariance is easily implemented in the lottery machine for each particular configuration of coin toss outcomes. Since each coin can be relabeled as $\mathrm{H}$ or $\mathrm{T}$ independently of 
the others, there will always be a relabeling that maps any particular configuration to any other. Thus each has an equal chance.

That is not quite enough to assure that the infinite lottery machine favors no particular number. For each number outcome corresponds to an infinite set of the configurations. Ideally we should like to show that a single relabeling of the randomizers can convert the entire set that returns outcome number $N$ to the entire set that returns outcome number $N$ '. A little reflection shows that no single relabeling can do this.

There is something enough close to it. Partition the set of configurations that return a definite number according to the row in which the first encoded number appears. Consider one member of the partition: those for which the first encoded number outcome appears in row $M$. That partition is further divided according to whether the number outcome is $1,2, \ldots$ The set of outcomes in this partition that returns number $N$ can be mapped onto the set that returns number $N$ : all that is needed is a relabeling of the randomizers in row $M$ alone so that the row now encodes $N^{\prime}$. All the other rows are left unchanged.

Hence, given that the first encoded number appears in row $M$, label invariance assures us that any of $1,2, \ldots$ are chosen without favor. This lack of favor is unaffected by which row number $M$ happens to be the first row that encodes a definite number. Hence the lottery as a whole is choosing its outcome without favoring any particular number.

\subsection{Nonmeasurable Outcomes}

The probability measure induced by (9) assigns a probability measure to many of the outcomes. For example, according to it, there is a probability 1 of there being infinitely many $\mathrm{H}$ in the array overall. However, outcomes of interest to us are almost all of the character of infinitely many trials of a zero probability process. They turn out to be nonmeasurable in this induced measure.

This lottery machine may fail to operate successfully if there is no row among the infinitely many that encodes a finite number. To demonstrate that success is a nonmeasurable outcome, we calculate the probability of success in two ways; and get two different answers.

First, we calculate by rows and find that success has zero probability. The probability that the first row has any particular combination of $\mathrm{H}$ and $\mathrm{T}$ is just $1 / 2^{\infty}=0$. Since there are only a countable infinity of finite numbers, the probability that the first row encodes any finite number 
at all is a countably infinite sum of these zeros, that is, zero. Hence, with probability one, the first row does not encode a finite number. The same follows for any nominated row. Since the row outcomes are probabilistically independent, the probability that no row encodes a finite number is just the product of these infinitely many ones, which is one. That is, there is probability zero of a row encoding a finite number in the array.

Second, we calculate by columns and find the probability of success is one. Consider the outcome HTTTTTT.... which encodes the number 1; and the truncated rows that appear in the first $\mathrm{N}$ columns. If this outcome is present in the array, then its first $N$ terms will appear in at least one of the truncated rows. The probability that some nominated row does not contain the truncated number is $\left(1-1 / 2^{N}\right)<1$. Since the row contents are probabilistically independent, the probability that the truncated number is in none of the infinitely many truncated rows is $\left(1-1 / 2^{N}\right)^{\infty}=0$. That is, the truncated number appears with probability one in at least one of the truncated rows of the first $N$ columns. Now take the limit as $N$ goes to infinity. This unit probability is preserved in the limit. With probability one, the row encoding 1 appears at least once in the array. The lottery machine is successful if any row encodes any finite number. Therefore the probability of success is greater than the probability of any row encoding just the number 1 . Therefore the probability of success is one.

By analogous reasoning, we find other nonmeasurable outcomes. The presence of a row encoding any particular finite number in the array is nonmeasurable. To see this, consider the rectangle of the first $M$ rows and $N$ columns, where $M=2^{N} K$ and the constant $K$ fixes the side ratio of the rectangle. The probability that the first $N$ terms of a row that encodes some nominated finite number is not a truncated row in the rectangle is $\left(1-1 / 2^{N}\right)^{M}=\left(1-1 / 2^{N}\right)^{2^{N} K}$. The limit of this probability as the size of the rectangle grows infinitely large is $\mathrm{e}^{-K}$. Hence the probability of at least one row encoding the nominated finite number is $\left(1-\mathrm{e}^{-K}\right)$. Since $0<K<\infty$, this probability can have values anywhere from 0 to 1 . It follows that the outcome is nonmeasurable.

\subsection{Reading the Outcome by a Supertask}

The process specified can select a natural number without favoring any. However it does not communicate the number. A lottery machine operator must search the array for the first row 
that encodes a finite number, both to recover the number and to learn if the operation was successful. This can be done by a supertask for which it is convenient to encode the outcome " 0 " by a row that is all $\mathrm{H}$. In the supertask, the operator reviews a square of the randomizers in the first $N$ rows and $N$ columns at time $\mathrm{t}=-1 / N$. The operator checks for a row that begins with $\mathrm{T}$ or contains somewhere within it the pair ...TH... Any such row is marked. Infinitely many squares of size $N=1,2,3, \ldots$ are checked at times $\mathrm{t}=-1,-1 / 2,-1 / 3, \ldots,-1 / N, \ldots$ Upon completion of the supertask at $\mathrm{t}=0$, all rows that fail to encode a natural number have been marked. The only unmarked rows are those that encode a number. The outcome is read as the number encoded by the first unmarked row. A second supertask is needed to search through the infinitely many rows for this unmarked row, since none of the rows may end up as unmarked.

\subsection{How Exotic is this Infinite Lottery Machine?}

This infinite lottery machine is the least exotic of those considered above, in so far as it does not require a supertask for its outcome number to be selected. While the idea of infinitely many, two-state randomizers may seem fanciful, they can be implemented in our universe, if standard modern cosmology is correct. If space is infinite in volume and roughly uniformly filled with galaxies, the individual galaxies can function as randomizers. Those with an even number of stars are "H"; and those with an odd number "T." Any labeling scheme can be used to organize the galaxies into the array. Since the outcomes are for each galaxy are independent, the choice will not matter. ${ }^{19}$

Instantiated as a galactic infinite lottery machine, we can ask what inductive support is accrued to such outcomes as: the lottery succeeds in selecting a number; the number has any value we specify, given that the lottery succeeds. These questions are meaningful in our universe. We do not need to transport ourselves in imagination to a neighboring, fictional universe.

19 The complication of the example, as a referee has reminded me, is to find a precise enough stipulation of what constitutes a galaxy and the count of the stars it contains. An easier course is to divide space into large volumes of equal size and assign an " $\mathrm{H}$ " or " $\mathrm{T}$ " according to whether the net charge of the volume is even or odd, when measured in units of electron charge. 
The first weakness of this design of infinite lottery machine is that the successful operation of the infinite lottery machine is not assured, but is nonmeasurable in the probability measure supplied by the randomizers. The best we can say, then, is that success is possible. That success is probabilistically nonmeasurable should not be confused with the dire case of a zero probability outcome. For a zero probability outcome, we expect no successes in many repeated trials. For the nonmeasurable outcome of success, different limiting procedures yield probabilities everywhere from 0 to 1 . In repeated trials, we may have successes. The success rate, however, will not stabilize to a limiting value.

The second weakness is that learning the number selected requires a supertask; and for that we do need to voyage to a neighboring, fictional universe.

\section{Conclusion}

How best can we design an infinite lottery machine? My answer is given in Section 11 above, employing the notion of possibility developed in Sections 4 to 7 . It may well not be the best answer possible. I have no proof of optimality. Might readers like to take up the enjoyable challenge of finding a better design?

\section{References}

Applegate, M. J.; Thomas, O.; Dynes, J. F.; Yuan, Z. L.; Ritchie, D. A.; and Shields, A. J. (2015) "Efficient and robust quantum random number generation by photon number detection," Applied Physics Letters 107, 071106, 1-5.

Bartha, Paul (2004) “Countable Additivity and the de Finetti Lottery,” The British Journal for the Philosophy of Science, 55, pp. 301-321.

Benci, Vieri, Horsten, Leon and Wenmackers, Sylvia (2013) "Non-Archimedean Probability," Milan Journal of Mathematics, 81, pp. 121-51.

Bernadete, José (1964) Infinity: An Essay in Metaphysics. Oxford: Clarendon.

Blackwell, David and Diaconis, Persi (1996) “A Non-Measurable Tail Set,” pp. 1-5 in T. S Ferguson, L. S. Shapley and J. B. McQueen, eds., Statistics, Probability and Game Theory: Papers in Honor of David Blackwell. Hayaward, CA: Institute of Mathematical Statistics. 
Davies, E. B., (2001) “Building Infinite Machines,” British Journal for the Philosophy of Science, 52, pp. 671-682.

de Finetti, Bruno (1972) Probability, Induction and Statistics. London: John Wiley \& Sons. Earman, John (manuscript) "Additivity Requirements in Classical and Quantum Probability"

Halmos, Paul R. (1973) “The Legend of John Von Neumann,” The American Mathematical Monthly, 80, pp. 382-394.

Hansen, Casper Storm (2016) “On Fair Countable Lotteries.” Philosophical Studies. Published online.

Jaynes, Edwin T. (2003) Probability Theory: The Logic of Science. Cambridge: Cambridge University Press.

Johnson, Walter R. (2007) Atomic Structure Theory: Lectures on Atomic Physics. Berlin: Springer.

Kadane, Joseph B. and O'Hagan, Anthony (1995), “Using Finitely Additive Probability: Uniform Distributions on the Natural Numbers," Journal of the American Statistical Association, 90, pp. 626-631.

Kadane, Joseph B,. Schervish, Mark J. and Seidenfeld, Teddy (1986) "Statistical Implications of Finitely Additive Probability" in Bayesian Inference and Decision Techniques. Amsterdam: Elsevier Science Publishers, pp. 59-76.

Kerkvliet, Timber (2016) “The Supertask of an Infinite Lottery,” Logique et Analyse. Vol. 59, No. 236, pp. 415-25. Preprint: http://arxiv.org/abs/1511.08182v1.

Ma, Xiongfeng; Yuan, Xiao; Cao, Zhu; Qi, Bing; and Zhang, Zhen, (2016) “Quantum random number generation," npj Quantum Information, 2, 16021, 1-9.

Manchak, John and Roberts, Bryan W., (2016) "Supertasks", The Stanford Encyclopedia of Philosophy (Summer 2016 Edition), Edward N. Zalta (ed.), http://plato.stanford .edu/archives/sum2016/entries/spacetime-supertasks/

McCall, Storrs and Armstrong, David M. (1989) “God's Lottery," Analysis, 49, pp. 223-224.

Norton, John D. (1999) "A Quantum Mechanical Supertask" Foundations of Physics, 29, pp. 1265-1302.

Norton, John D. (2008) "The Dome: An Unexpectedly Simple Failure of Determinism" Philosophy of Science, 75, pp. 786-98. 
Norton, John D. (2011) “Challenges to Bayesian Confirmation Theory," Philosophy of Statistics, Vol. 7: Handbook of the Philosophy of Science. Prasanta S. Bandyopadhyay and Malcolm R. Forster (eds.) Elsevier.

Pruss, Alexander R. (2014) "Infinitesimals are Too Small for Countably Infinite Fair Lotteries," Synthese, 19, pp. 1051-57.

Sommerfeld, Arnold (1923) Atomic Structure and Spectral Lines. Trans. Henry L. Brose. London: Methuen.

Spielman, Stephen (1977) "Physical Probability and Bayesian Statistics," Synthese, 36, pp. 23569.

Van Strien, Marij (2014) “The Norton Dome and the Nineteenth Century Foundations of Determinism," Journal for General Philosophy, 45, pp. 167-85.

Weintraub, Ruth (2008) "How Probable Is an Infinite Sequence of Heads? A Reply to Williamson," Analysis, 68.3, pp. 247-50.

Wenmackers, Sylvia and Horsten, Leon and (2013) “Fair Infinite Lotteries," Synthese, 190, pp. $37-61$.

Williamson, Jon (1999) “Countable Additivity and Subjective Probability,” British Journal for the Philosophy of Science, 50, pp. 401-16.

Williamson, Timothy (2007) "How Probable is an Infinite Sequence of Heads," Analysis, 67.3, pp.173-80. 


\title{
Correction to John D. Norton "How to Build an Infinite Lottery Machine"
}

\author{
John D. Norton ${ }^{1}$ \\ Department of History and Philosophy of Science \\ University of Pittsburgh \\ jdnorton@pitt.edu http://www.pitt.edu/ jdnorton \\ and \\ Alexander R. Pruss \\ Department of Philosophy \\ Baylor University \\ alexander_pruss@baylor.edu http://alexanderpruss.com
}

\begin{abstract}
This note corrects an error in John D. Norton, "How to Build an Infinite Lottery Machine."
\end{abstract}

Norton (2018) examines physical devices intended to realize an infinite lottery machine that can select from a countable infinity of outcomes without favoring any. Readers will be helped by consulting it before proceeding. Successful proposals seem to require exotic processes, such as supertasks or probability zero processes. The most promising of the proposals considered in Norton (2018) was one that employs an infinite array of coin tosses. Section 11.3 of Norton (2018) argues that its successful operation is a nonmeasurable event in the background probability measure of the coin flips of the array. The argument is fallacious and successful operation is provably a probability zero outcome.

This zero probability of success makes the machine of Section 11 less interesting and comparable in significance to the pointer on a dial machine of Section 2.3, since both now only succeed with probability zero. More significantly its failure means that the investigation has not found a design for an infinite lottery machine that employs only finite randomizers, like binary

${ }^{1}$ JDN: I thank Alex Pruss for drawing my attention to the problems in Section 11.3. 
coin flips, that choose among a finite number of outcomes. This is so even when we employ infinitely many finite randomizers and accelerate our processes with supertasks. It makes more plausible the conjecture that no such design can succeed, except with probability zero. In light of this change, the most promising design among those of Norton (2018) is now the exotic quantum mechanical lottery machine of Section 10 . Its primitive process is, by supposition, already a randomizer that selects among an infinity of outcomes.

The details of the fallacy in Section 11.3 are not especially illuminating, but are included here for completeness. The argument by rows arrives at the correct conclusion of zero probability in classical, countably additive probability theory. Moreover, the argument shows that the event of successful operation is measurable. Its measure is constructed from the measurable outcomes of individual coin tosses with complements, countable intersections and countable unions. There is no supposition of measurability that could figure in a reductio argument against measurability. Contrary to the suggestion of Section 11.3, no constructive argument within the probability calculus can yield a different probability for the outcome, unless the calculus is inconsistent. The arguments by column and by rectangles of $M$ rows and $N$ columns fail. Each is based on a limiting process that should produce a set of all arrays that do not contain the row HTTTTT... The limiting process in each produces only a proper subset of this set. A counterexample for the "by columns" argument is the array:

НTHНHНH...

НТТНННН...

НТТТННН...

НTТТТНН...

This array does not contain the row HTTTTT... but it is not in the limit set. Analogous counterexamples can be found for the argument by $M \times N$ rectangles.

If the number of rows in the array is uncountable, then the event of the array having at least one row that encodes a counting number is nonmeasurable. ${ }^{2}$ However identifying the first row encoding a counting number is problematic. The notion of such a first row requires a

2 This follows from the fact proved by Norton in Section 11.3 that the probability that a particular fixed row contains the encoding of a counting number is zero together with the Proposition in Pruss (2017). 
nonconstructive well-ordering of the uncountable set of rows, and additionally one would need a process that that traverses that uncountable set in that order.

\section{References}

Norton, John D. (2018) "How to Build an Infinite Lottery Machine," European Journal for Philosophy of Science, this issue.

Pruss, Alexander R. (2017) “Uncountable Independent Trials,” blog post, http://alexanderpruss.blogspot.com/2017/08/uncountable-independent-trials.html. 